\title{
Interactive comment on "Minimizing the impact of vacating instream storage of a multi-reservoir system: a tradeoff study of water supply and empty flushing" by Chia-Wen Wu et al.
}

\section{Anonymous Referee \#1}

Received and published: 21 September 2020

\section{GENERAL COMMENTS}

This paper explores the feasibility of empty flushing in a two-reservoirs system, minimizing the impact of the operation on the multiple use of water storage (i.e., municipal, agricultural, industrial, and hydropower supply). The reservoirs are located in SW Taiwan. Due to siltation, the larger one (Tsengwen Reservoir) has lost about $30 \%$ of its original storage capacity $(630 \mathrm{Mm} 3)$ in the 45 years following dam closure (1973). The smaller one (Wushanto Reservoir), if properly managed, could satisfy local water demand when flushing Tsengwen Reservoir. The general subject of adopting empty flushing to recover reservoir storage, and the specific topic of optimizing multi-purpose

Printer-friendly version

Discussion paper 
systems comprising several reservoirs deserve in my opinion the interest of the international scientific literature dealing with water resources management. I think that the manuscript could be significantly improved by considering comments and suggestions provided below.

\section{SPECIFIC COMMENTS}

1. In my opinion, the paper is overly long. To improve its readability, I suggest to shortening or moving to Appendixes or Supplementary Material sections and paragraphs of minor importance relatively to the main objective of the study. Examples are reported below.

- Table 1 might be moved to Appendix/Supplementary Material.

- The estimation of parameter psi of Equation 1 (P35-P38), including data from further reservoirs, might be moved to Appendix/Supplementary Material.

- The sensitivity analysis (P45-46) might be moved to Appendix/Supplementary Material. By the way, the linear variation of the desilting volume with psi could have been expected, due to the structure of Equation1.

- The 2D simulations of sediment transport throughout the drawn-down reservoir during empty flushing (P 47-48) can be moved to Appendix/Supplementary Material. From the one hand, I think that the efforts made by the Authors to validating the adopted psi value are commendable. However, the proper presentation of these simulations would require additional space, overloading the paper.

2. Section Results and Discussion contains several elements related to the description of the investigated system and of the adopted methodology. I suggest moving these paragraphs to the Methods section. Examples are reported below.

Printer-friendly version

- Water demand of the system and inflow to Tsengwen Reservoir (P29, Figure 5) are not results, and can go to the subsection describing the case study. 
- The scheme of the system (Figure 6) is not a result, and can go to the subsection describing the case study.

- The modified balancing curves (P31, Figure 7) are not results, and would be moved to the Methods section.

- The methodology to assessing the impact of empty flushing on the short-term water supply (P38-39) is not a result, and would be moved to the Methods section.

- The refinement through Equations 12 and 13 (P41) is not a result, and would be moved to the Methods section.

3. Partly connected to previous point 2 : though the adopted methodology could be extended to multi-reservoirs systems, it was developed (I would say "tailored") on the tworeservoirs study case. I therefore suggest to fully describing the investigated system in a dedicated subsection, and to describing the adopted methodology with specific reference to the case study. Later on, in the Discussion section, the Authors can comment on the possible extension to different multi-reservoirs systems. In the current version of the manuscript, information concerning the investigated system is fragmented over different paragraphs, thus confusing the reader.

4. The calibration of the model throughout the period 1974-2009 and its validation in the following years (2010-2017 - Par. 3.7) is rather unclear to me: I expected some comparison of simulated vs observed data, but I did not noticed it. Perhaps this point might be clarified.

5. The proposed strategy poses some risks (shortage at least, but also hydraulic and environmental issues to the river section below Tsengwen Reservoir). In contrast, the predicted contribution to desilting Tsengwen Reservoir is low (Figure 18). Accordingly, current management adopts hydro-suction, downstream settling, and removal by flood spilling (P24-25). Moreover, the capacity to inflow ratio for Tsengwen would seem very high (in the range $4-5$, depending on the adopted storage, original or current),

Printer-friendly version

Discussion paper 
but my estimate of CIR can be affected by wrong inflow data provided in Par. 2.4 (see Technical Corrections below). I think that these arguments would be properly addressed and deeply discussed in the revised version of the paper.

6. The environmental impact of empty flushing has, in my opinion, marginal relevance in the proposed strategy. Rather than presenting it as automatically addressed (Par. 2.3, and particularly P23, L18-20), I would comment it the Discussion section (possibly including more recent references) as a potential source of further constraints. In fact, when considering the impact of sediment flushing on downstream biotas, limits on suspended sediment concentration and dissolved oxygen (as well as on streambed aggradation) should be accounted for.

\section{TECHNICAL CORRECTIONS}

P3, L5: "extraordinary water quality" is unclear, please rephrase.

P12, L20: "capacitated" is unclear, please rephrase.

Figures writing is frequently too small and could be enlarged to improve readability (see Figures 1, 5, 6, and 13).

P19, L6: in order to avoid confusion with sediment, water can be specified before "volume".

P19, L9: did you mean refill instead of "fulfill"?

P26, L13: I would remove "experimental setup".

P26, L20: I think that $120 \mathrm{Mm3}$ annual inflow is too small, suggest checking this (very important) parameter (see previous point 5).

P28, L13: replace "result" by results.

Printer-friendly version

P36, L23-24: how did you get volumetric concentrations? The adopted sediment density is not specified. 
Interactive comment on Hydrol. Earth Syst. Sci. Discuss., https://doi.org/10.5194/hess-2020258, 2020.

Interactive

comment 